\begin{abstract}
Background: Understanding patients' experiences and preferences for health care is important to inform how to provide best-practice, patient-centred care. This study aimed to explore the perceptions of patients who have been recently diagnosed with Type 2 diabetes regarding nutrition care received from dietitians.
\end{abstract}

Methods: Ten individuals recently diagnosed with Type 2 diabetes participated in three individual semi-structured qualitative telephone interviews: at baseline, then at three and six months after recruitment. Data were analysed using content analysis at each time point and meta-synthesis of findings over time.

Results: Participants’ initial interactions with dietitians were challenging and overwhelming due to the instructional nature of consultations. Many participants questioned the use of dietary guidelines to inform nutrition care, because this was not adapted to the individual. Some participants valued receiving education on topics such as label reading and serving sizes, however others thought that the nutrition care was rushed and overly directive. Very few participants perceived an ongoing relationship with a dietitian would be useful, and limited interaction was planned beyond six months after diagnosis.

Conclusion: These findings suggest there is considerable opportunity for dietitians to enhance the nutrition care provided to patients with Type 2 diabetes. Tailoring of dietary guidelines to individuals, utilising supportive counselling styles, and focusing on open communication in consultations that facilitates ongoing, useful care for patients may help patients with Type 2 diabetes achieve and maintain healthy dietary behaviours. 


\section{The nutrition care needs of patients newly diagnosed with Type 2 diabetes: Informing dietetic practice}

\section{Introduction}

Type 2 diabetes is a leading cause of morbidity and mortality in Australia, accounting for over $2.3 \%$ of all direct health care expenditure (1). Over $5 \%$ of the Australian population have been diagnosed with Type 2 diabetes, and its prevalence is increasing faster than any other chronic condition (2). The health outcomes of patients with Type 2 diabetes are significantly influenced by dietary behaviours (3). Improvements in dietary behaviours of patients with Type 2 diabetes are associated with reduced risk of complications and improved glycaemic control $(3,4)$. As a result, supporting patients to achieve and sustain healthy dietary behaviours is a fundamental component Type 2 diabetes management by primary health professionals $(5,6)$.

Dietitians are financially supported in Australia to provide nutrition care to patients with Type 2 diabetes through the Medicare Chronic Disease Management (CDM) Program (7). Dietitians are the third largest allied health providers within the Program, and the rate of dietetic service utilisation has increased annually over the past decade. Over 300,000 dietetic consultations were conducted in Australia through the CDM Program in 2013 (8). Dietary intervention studies have demonstrated that dietitians can support patients with Type 2 diabetes to significantly improve their glycaemic control, body composition and risk factors for future complications $(3,9)$. However, patients with Type 2 diabetes generally do not utilise all dietetic consultations available to them through the CDM Program, despite more frequent consultations being associated with better outcomes (10). Extrapolating data on the prevalence of Type 2 diabetes in Australia and the rate of dietetic service utilisation, fewer 
than one in three individuals with Type 2 diabetes consult a dietitian once each year $(1,8)$. This suggests that patients' engagement with dietitians in the Australian primary health system is less than ideal $(3,9)$. A better understanding of patient experiences of nutrition care received from dietitians is needed to guide the profession on ways to improve health care practices.

Understanding patients' experiences and preferences for health care is important to inform how to provide best-practice, patient-centred care. Patient-centred care is an approach to healthcare that meets the specific needs, values and beliefs of patients (11) and is regarded as imperative to the optimal management of chronic disease, including Type 2 diabetes $(12,13)$. In the context of nutrition, positive perceptions of care are likely to be important because of its association with increased patient comfort, increased behaviour change and improved patient outcomes (14). Health professionals who typically engage with patients on the topic of nutrition include general practitioners, practice nurses, diabetes educators and dietitians. Given the influence of dietary behaviours on the management of Type 2 diabetes, it is important that dietitians understand patients' perceptions of the nutrition care they receive. This will inform dietetic practice that may result in patients having more positive perceptions of their care, and identify strategies to increase patients’ engagement with dietetic services when required.

The data presented in this paper are a component of a larger study that explored how patients newly diagnosed with Type 2 diabetes adjusted to diagnosis, sustained dietary changes and interacted with primary health professionals (blinded for peer review). Overall, findings indicated that patients initially made wide ranging attempts to improve dietary behaviours, but experienced negative emotions over time from the restraint required to maintain a healthy diet. Patients were confused by the amount of conflicting dietary advice they had received, 
and reported both positive and negative experiences with health professionals. Participants made regular, specific remarks about their ongoing relationships with health professionals, and how the support received from dietitians related to their experience of dietary change. In order to capture these valuable insights, this study reports on the perceptions of patients who have been recently diagnosed with Type 2 diabetes regarding nutrition care received from dietitians. Specifically, the study explored the nutrition care needs of newly diagnosed patients over time, and their views on how dietitians can best support long-term maintenance of dietary change.

\section{Methods}

The methodology of the larger study has been described elsewhere (blinded for peer review). Briefly, the study utilised a longitudinal, interpretive, qualitative design to explore the perceptions and experiences of patients who self-reported to be recently diagnosed with Type

2 diabetes. The research design was informed by guidelines for nutrition and dietetics studies that utilise qualitative and longitudinal approaches $(15-18)^{19}$. To be eligible, patients had to self report they were diagnosed with Type 2 diabetes in the previous four months. A purposive sample of individuals was recruited via e-newsletters and social media pages of Australian diabetes organisations. Potential participants were provided with a plain language information sheet, prior to providing consent and arranging an initial interview. The study was approved by the blinded for peer review Human Research Ethics Committee (reference number PBH/09/14/HREC).

Each participant was involved in three individual semi-structured telephone interviews: at baseline (i.e. within 4 months of diagnosis), and then at 3 and 6 months after recruitment into the study. A semi-structured interview guide was developed for each round of interviews. Specific questions were asked about the care received from health professionals, such as 
“describe your interactions with health professionals regarding food and healthy eating” and "at this moment, how could health professionals be most helpful in supporting you in healthy eating? Elaboration probing such as “can you provide further information about that?”, clarification probing such as “you mean receiving recipes?” and attention probing such as "that was an interesting experience, can you explain more" were used at the discretion of the interviewer to gain a deeper understanding of the participants’ perceptions. Interview questions at 3 months and 6 months were modified from the initial baseline interviews in order to investigate emerging findings (19).

All interviews were conducted by one investigator (RD), who had experience in the conduct of qualitative interviews, but was not a health professional; and all participants were made aware of the investigator's background. Interviews were 30-60 minutes in length and were digitally recorded and transcribed verbatim. Immediately after the interviews, an entry was made in a reflective journal that outlined the overall impressions of the interview, and key responses for each question. Data collection and analysis were conducted concurrently, whereby recruitment, data collection and analysis continued until data saturation was achieved. In practical terms, saturation was considered to have been reached when no new preliminary categories were detected as interviews progressed (20).

A two-step data analysis process occurred for this study. Firstly, content analysis was used to analyse the verbatim transcripts at each time point because it allows an in-depth understanding of participants' nutrition care needs $(21,22)$. Analysis of verbatim transcripts involved an iterative process of reading the transcripts to become familiar with the data, generating initial sub-categories, and then searching for, reviewing, and finally labelling the categories $(21,22)$. Triangular analysis was conducted by two investigators (LB and RD) by independently generating initial sub-categories, and then comparing and discussing to reach 
agreement. Secondly, a meta-synthesis approach was undertaken to synthesise and interpret the data across the three time points (23). The iterative analytic process involved extracting the sub-categories, then interpreting, merging and expanding the sub-categories in order to identify higher order categories that transcend findings from each time period. Regular meetings with the whole research team ensured ongoing examination of the emerging findings and that the finalised three categories, each with three sub-categories, accurately reflected the data for transferability and credibility.

\section{Results}

Ten individuals recently diagnosed with Type 2 Diabetes participated in the study, each completing three interviews. The participants included 3 men and 7 women, and their ages at the time of diagnosis ranged from 27-74 years. The analysis revealed three categories and sub-categories which are displayed in Table 1.

\section{INSERT TABLE ONE ABOUT HERE}

\section{Receiving Directive Nutrition Care is Overwhelming and Not Helpful}

The first category related to participants' initial experience of having an appointment with a dietitian and receiving nutrition care. Eight of the ten participants had at least one appointment with a dietitian. The two participants who did not have contact with a dietitian reported to (i) be confident with their dietary intake and not require support in this area; and (ii) be unclear about the benefit of receiving support from a dietitian. For the participants who received nutrition care, this interaction generally occurred soon after diagnosis, following a referral from the participants’ General Practitioner. The initial interactions with dietitians 
were often viewed as challenging and overwhelming, because of the directive, instructional nature of the consultation.

“A few days after I was diagnosed I went to a dietitian and she laid it all out. I wasn't impressed. This woman was just there to purposely lay down a diet, to put me in line and show me this, that, this that.” (Participant 1, Female, Aged 66 years, Interview 1) "The dietitian, I found her a bit hard going at the start. She put a bit of pressure on me straight away... 'what are you going to give up? What three things are you going to give up or change?'...I'd had a nice lead in with the [diabetes] educator and then all of a sudden I saw the dietitian and she was laying down the law.” (Participant 2, Male, Aged 57 years, Interview 1)

Participants displayed variable views about the helpfulness of their initial interaction with dietitians. Some participants found the nutrition care they had received assisted them to feel confident about the ideal foods to eat, whereas other participants reported that their experiences with a dietitian did not align with their expectations.

"I think the first time with the dietitian is necessary, they can give you the whole idea about which foods you can eat and which foods to avoid.” (Participant 5, Female, Aged 27 years, Interview 3)

"I've seen the dietitian, and I kept a food diary. I mean they do try and she's a very nice dietitian, but I think sometimes more is needed.” (Participant 1, Female, Aged 66 years, Interview 2)

\section{Contribution of Dietary Guidelines in Supporting Dietary Change}

The second category related to participants' views on the content of nutrition care they received from dietitians. Across the three interviews, some participants reported that information provided by dietitians increased their ability to achieve and sustain a healthy 
dietary intake. This was achieved through greater confidence in which foods are ideal to eat, and greater skills in preparing, accessing and selecting healthy foods.

"I've got to the stage now where I don't think too much about the diet because you're just in a habit of eating healthy meals so I know now what I should be eating, and what are sometimes foods.” (Participant 2, Male, Aged 57 years, Interview 2)

"They [dietitian] were there to help me...how to read product labels, how to do your serving size right, they are helping so that I can make better choices or find alternative foods.” (Participant 5, Female, Aged 27 years, Interview 1)

However, participants tended to question the relevance of nutrition care informed by dietary guidelines (such as the Australian Guide to Healthy Eating), because this care and the guidelines were not perceived as being individually tailored to their unique nutrition requirements.

"I like fruit, but they [dietitians] reckon you can only have 2 pieces a day, and I think that's not enough for me, I need more. So yeah, if you go by their guidelines it doesn't always work. You've got to work out what your body needs.” (Participant 1, Female, Aged 66 years, Interview 2)

“The guidelines are all Western foods. If I eat Western foods all week I think I will die! I really don't even know how to cook them...so I just make up my own menu for myself." (Participant 5, Female, Aged 27 years, Interview 2)

After initially being educated about the content of the dietary guidelines, participants reported high exposure to conflicting dietary messages, which caused confusion about the ideal foods to eat.

"I kept finding so much controversy it made my head spin...they [dietitians] say you have protein, you have carbs, and you have your veggies and stuff. Then you will get this 
other advice, no meat, no animal protein, no none of this, no oils... Who is right? Who is wrong? Which way do you go? I found it confusing.” (Participant 2, Male, Aged 57 years, Interview 1)

"In earlier stages they [a dietitian] said to me watermelon is out, now in the last book I got from Diabetes SA it says they can't wait for summer because we can all splurge into the watermelon...so I'm getting a bit confused.” (Participant 7, Female, Aged 72 years, Interview 2)

\section{Desiring More Individualised Care}

The third category encompassed participants' views on their relationship with dietitians, as well as dietitians’ delivery of nutrition care. During the six months following recruitment into the study (up to 10 months after diagnosis), two participants did not interact with a dietitian, two participants had one consultation with a dietitian, five participants had two consultations with a dietitian, and one participant had three consultations with a dietitian. Some participants perceived that dietitians were genuinely supportive when providing nutrition care, but many reported feeling rushed and not heard in consultations.

“It wasn't like she was looking at me or judging me on what I should be eating. If I could photocopy her millions of times around Australia I would.” (Participant 4, Female, Aged 30 years, Interview 2)

“You're talking and they go 'your half hour's up, I have another patient waiting'...Nobody has got the time to sit and take the time to talk. I find that very frustrating and annoying. You just start to say something and then you've got to leave." (Participant 1, Female, Aged 66 years, Interview 3)

Based on their experiences of receiving nutrition care, participants identified opportunities for dietitians to enhance the care provided to patients, which focused on the delivery of nutrition 
care in consultations. Specifically, participants valued genuine relationships, tailored advice, open communication and sufficient time in consultations.

"For a relationship with the patient...it's vitally important to treat the patient like a person, and not a name on a form.” (Participant 9, Male, Aged 69 years, Interview 3) “Don't put everyone under the same banner. Be more individualised and try to sit down and take time, talk, work things out. Don't try and fix me the way you fixed the ones before, we are all different. You can't just go on assumptions. It doesn't work." (Participant 1, Female, Aged 66 years, Interview 3)

Very few participants perceived that an ongoing relationship with a dietitian would be useful, and limited interaction was planned for the future. Most participants thought that further interactions with a dietitian would not be useful, because of the content and delivery of the initial one or two consultations. Interestingly, this perception was consistent for participants who were satisfied with the nutrition care they had received from dietitians, as well as participants who felt they may benefit from additional general support in improving their dietary behaviours.

“It's worked out pretty well. I can't see myself ever drifting back eating the same way as I was...I'll just continue to eat as healthily as I can, there's no benefit from seeing her [the dietitian] again.” (Participant 2, Male, Aged 57 years, Interview 3) "The first time with the dietitian is necessary, because they can give you a whole idea about which foods to eat, and which foods to avoid...then I don't think it's necessary anymore, I don't think we need them anymore.” (Participant 5, Female, Aged 27 years, Interview 3)

\section{Discussion}

This study contributes new information on patients' perceptions of receiving nutrition care 
from dietitians after being diagnosed with Type 2 diabetes. Three categories were generated from the data; 1. Receiving Directive Nutrition Care is Overwhelming and Not Helpful; 2. Contribution of dietary guidelines in supporting dietary change; and 3. Desiring more individualised care. This study is important because it provides a better understanding of patients' experiences of receiving nutrition care from dietitians and their nutrition care needs following diagnosis of Type 2 diabetes. The study also informs dietetic practice by identifying strategies which may improve care delivery and enhance patients' experiences and engagement with dietitians. These experiences have the potential to influence dietary behaviours and health outcomes in type 2 diabetes $(3,4)$, and therefore warrant attention by the dietetic profession.

Both the first and the third category reflect patients’ desire to be treated as individuals. Patient-centred care focuses on patients' needs, values, beliefs and preferences (11) and is regarded as imperative to the optimal management of chronic disease, including Type 2 Diabetes $(12,13)$. The experiences of participants in this study suggest that patient-centred care was not always achieved and warrants improvement. Specifically, some participants’ experience of receiving nutrition care from a dietitian was reported as challenging because of the directive, instructional nature, which did not always align with their preferences for care. In the larger study, participants also reported variable perceptions of dietary change (blinded for peer review) and held different views about the helpfulness of dietitians in this process. This variability suggests that a single approach to providing nutrition care may not be appropriate, and that dietitians should enhance their capacity to provide patient-centred, flexible and tailored nutrition care. This may be achieved through additional training in patient-centred lifestyle counselling. There is emerging recognition of the value of understanding patients’ preferences in other areas of diabetes care $(24,25)$. Therefore, tailoring the delivery of nutrition care (including content, counselling style and length of 
consultation) based on patients' individualised preferences and needs may facilitate positive health care experiences ${ }^{(26) .}$ In turn, this may increase the likelihood of patients maintaining ongoing relationships with dietitians, and making sustained improvements to their dietary behaviours.

Participants questioned the relevance of nutrition care that mirrored the national dietary guidelines because it was perceived as not being tailored to their individual nutrient requirements. The Australian Guide to Healthy Eating is intended to provide broad principles for dietary intake (such as food groups and portion sizes), and was developed to allow flexibility in patterns of eating $(26)^{(27) .}$ This suggests that patients' experiences of receiving nutrition care may be improved if dietitians deliberately demonstrate in consultations how the dietary guidelines are being tailored to the patient. This could be achieved by incorporating the patients’ individual food preferences, daily family and logistical circumstances and dietary goals to the advice provided. These actions may also provide opportunities to acknowledge the likelihood of patients receiving conflicting dietary messages after a dietetic consultation. This approach aligns with the concept of shared-decision making, whereby patients and health professionals intentionally cooperate to make decisions about health care $(27)^{(28)}$. Shared-decision making has been shown to enhance patients' knowledge and confidence in chronic disease management ${ }^{(27)(28)}$, which is particularly relevant for making sustained improvements to dietary behaviours. Given that participants in the present study perceived the nutrition care as overly directive, shared-decision making provides an opportunity to allow patients to have equal input into the consultation outcomes. However, one Canadian study identified the adoption of shared-decision making in dietetic practice to be low $(28)^{(29)}$, and warrants greater emphasis when providing nutrition care. 
Participants valued the support that dietitians provided regarding skills to assist with food selection and preparation, including label reading and recipe modifications. Other primary health professionals such as doctors and nurses have reported low self confidence in their knowledge of specific foods and their nutrient composition, as well as poor skills in nutrition label reading $(29,30)^{(30-31)}$. This highlights a likely point of difference in the nutrition care provided by dietitians compared with other health professionals, which is important given the confusion reported by patients in a previous study on the role of different Australian health professionals in providing nutrition care $(31)^{(32)}$. Given the potential influence of increased food skills on dietary behaviours for individuals with Type 2 diabetes ${ }^{(33)}$ the role of dietitians in supporting individuals is notable and warrants further emphasis.

Participants reported that feeling genuinely supported in consultations, and not feeling rushed, were desirable components of nutrition care from dietitians. Similar factors have been reported to influence patients' experiences of health care in other Australian population groups, such as new mothers, where their perceptions of their health care quality were reduced when they felt unsupported and hurried in consultations $(32)^{(34)}$. Collectively, this suggests that dietitians could enhance the delivery of their care by investing in patient relationships through more patient-focused consultations. One approach to providing patientfocused consultations involves promoting patient-participation $(33)^{(35)}$. Four attributes to patient-participation have been proposed, (i) having an established relationship, which is mutual, trusting, respectful and connected; (ii) surrendering power or control by the health professional, to facilitate equality; (iii) sharing information and knowledge, including obtaining patients' opinions, expectations and experiences; and (iv) having active mutual engagement in activities, by inviting, encouraging and supporting patients' input ${ }^{(33)(35)}$. Promoting these four attributes is consistent with the perceptions expressed in this study, suggesting there is opportunity for dietitians to enhance the nutrition care provided to patients 
by actively considering the attributes of patient-participation. Developing guidelines to support dietitians to provide patient-focused consultations is clearly an avenue for future research.

There are a number of strengths and limitations to the present study. This study has provided insights into opportunities for dietitians to improve the nutrition care provided to patients recently diagnosed with Type 2 Diabetes. The study also provided a conceptual understanding of nutrition care, but the extent to which it is appropriate for other conditions patient groups and countries is unknown. One interviewer for all interviews enhanced rapport building with participants, and increased consistency of data collection. It is possible that the recruitment involved a sampling bias, whereby individuals who had initial negative experiences with dietitians were more likely to take part in the study. Despite this limitation, the longitudinal nature of the study meant that participants were already recruited in to the study prior to experiencing most of the nutrition care that formed the basis of their experiences. Telephone interviews prevented the interviewer from observing non-verbal communication from participants. However, telephone interviews allowed for a heterogeneous sample from a diverse geographical location to be involved in the sample. In order to inform future work that provides practical steps dietitians can take to improve nutrition care, further research is warranted exploring dietitians’ perspectives on their current care and opportunities for improvement.

This study explored patients' experiences of dietary change and the support provided by dietitians. Participants’ initial interactions with dietitians were challenging and overwhelming due to the instructional nature of consultations. Many participants questioned the use of dietary guidelines to inform nutrition care, because this care was not perceived as being individually tailored to patients' unique requirements. Some participants valued receiving 
education on skills such as label reading, however others felt that the nutrition care was rushed, overly directive and lacking genuine support. These findings suggest there is considerable opportunity for dietitians to enhance the nutrition care provided to patients by demonstrating individual tailoring of dietary guidelines, utilising supportive counselling styles, and focusing on open communication in consultations.

Acknowledgements: The authors wish to thank the participants for sharing their views and experiences.

Funding: This study was supported by a Griffith University New Researcher Grant.

Conflicts of Interest: None declared. 


\section{References}

1. Australian Institute of Health and Welfare. (2007). Incidence and prevalence of chronic diseases. Canberra.

2. Australian Bureau of Statistics (ABS). (2012). Australian Health Survey: First Results. Canberra.

3. Coppell, K. J., Kataoka, M., Williams, S. M., et al. (2010). Nutritional intervention in patients with type 2 diabetes who are hyperglycaemic despite optimised drug treatment-Lifestyle Over and Above Drugs in Diabetes (LOADD) study: randomised controlled trial. BMJ 341.

4. Cooper, A. J., Schliemann, D., Long, G. H., et al. (2014). Do improvements in dietary behaviour contribute to cardiovascular risk factor reduction over and above cardioprotective medication in newly diagnosed diabetes patients? Eur J Clin Nutr, 68 11131118.

5. Diabetes Australia, \& Royal Australian College of General Practitioners (RACGP). (2008). Diabetes Management in General Practice 14th edition. Sydney.

6. Dietitians Association of Australia (DAA). (2011). Evidence Based Practice Guidelines for the Nutritional Management of Type 2 Diabetes Mellitus for Adults. Sydney.

7. Australian Government Department of Health and Ageing. (2015). Chronic Disease Management (CDM) Medicare Items (website) Accessed July 2015. from www.health.gov.au/main/publishing.nsf/Content/mbsprimarycarechronicdiseasemanagement

8. Cant, R., \& Ball, L. (2015). A decade of Medicare: the contribution of Private Practice Dietitians to chronic disease management and diabetes group services. Nutr \& Diet (Online early view) DOI: 10.1111/1747-0080.12175. 
9. Andrews, R., Cooper, A., Montgomery, A., et al. (2011). Diet or diet plus physical activity versus usual care in patients with newly diagnosed type 2 diabetes: the Early ACTID randomised controlled trial. Lancet, 378 129-139.

10. Spencer, L., O'Shea, M. C., Ball, L., et al. (2014). Attendance, weight and waist circumference outcomes of patients with type 2 diabetes receiving Medicare-subsidised dietetic services. Aust J Prim Health, 20 291-297.

11. McMillan, S., Kendell, E., Sav, A., King, M., Whitty, J., Kelly, F., \& Wheeler, A. (2013). Patient-centred approaches to health care: a systematic review of randomized controlled trials. Med Care Res Review, 70, 567-596.

12. Lusk, J. M., \& Fater, K. (2013). A concept analysis of patient-centered care. Nurs Forum, 48 89-98.

13. Holman, H., \& Lorig, K. (2000). Patients as partners in managing chronic disease. Partnership is a prerequisite for effective and efficient health care. BMJ, 320 526-527.

14. Maddigan, S., Majumdar, S., \& Johnson, J. (2005). Understanding the complex associations between patient-provider relationships, self-care behaviours, and healthrelated quality of life in type 2 diabetes: A structural equation modeling approach. Qual Life Research, 14 1489-1500.

15. Draper, A., \& Swift, J. A. (2011). Qualitative research in nutrition and dietetics: data collection issues. J Hum Nutr Diet, 24 3-12.

16. Fade, S., \& Swift, J. (2011). Qualitative research in nutrition and dietetics: data analysis issues. J Hum Nutr Diet, 24, 106-114.

17. Pilnick, A., \& Swift, J. A. (2011). Qualitative research in nutrition and dietetics: assessing quality. J Hum Nutr Diet, 24, 209-214.

18. Swift, J. A., \& Tischler, V. (2010). Qualitative research in nutrition and dietetics: getting started. J Hum Nutr Diet, 23 559-566. 
19. Thomson, R., \& Holland, J. (2003). Hindsight, foresight and insight: The challenges of longitudinal qualitative research. International Journal of Social Research Methodology, 6 233-244.

20. Strauss A, \& Corbin J. (2007). Basics of Qualitative Research: Techniques and Procedures for Developing Grounded Theory 3rd Edition. California: Sage Publications.

21. Graneheim, U. H., \& Lundman, B. (2004). Qualitative content analysis in nursing research: concepts, procedures and measures to achieve trustworthiness. Nurse Educ Today, 24 105-112.

22. Hsieh, H. F., \& Shannon, S. E. (2005). Three approaches to qualitative content analysis. Qual Health Research, 15 1277-1288.

23. Sandelowski, M., \& Barroso, J. (2007). Handbook for synthesizing qualitative research. New York: Springer Publishing Company, Inc.

24. Ahmann, A., Szeinbach, S. L., Gill, J., et al. (2013). Comparing Patient Preferences and Healthcare Provider Recommendations with the Pen Versus Vial-and-Syringe Insulin Delivery in Patients with Type 2 Diabetes. Diabetes Technol Ther, 16 76-83.

25. Purnell, T. S., Joy, S., Little, E., et al. (2014). Patient Preferences for Noninsulin Diabetes Medications: A Systematic Review. Diabetes Care, 37, 2055-2062.

26. Franz MJ, Boucher JL, Evert AB. (2014) Evidence-based diabetes nutrition therapy recommendations are effective: the key is individualization. Diabetes, metabolic syndrome and obesity : targets and therapy. 7:65-72.

27. Commonwealth of Australia. (2014). The Australian Guide to Health Eating. Melbourne.

28. Gionfriddo, M., Leppin, A., Brito, J., et al. (2014). A systematic review of shared decision making interventions in chronic conditions: a review protocol. Systematic Reviews, 3 38-45. 
29. Vaillancourt, H., Legare, F., Lapointe, A., et al. (2014). Assessing patients' involvement in decision making during the nutritional consultation with a dietitian. Health Expect, 17 545-554.

30. Crowley, J., Ball, L., Han, D., et al. (2015). Doctors’ attitudes and confidence towards providing nutrition care in practice: Comparison of New Zealand medical students, GP registrars and GPs J Prim Health Care, In Press.

31. Martin, L., Leveritt, M., Desbrow, B., et al. (2014). The self-perceived knowledge, skills and attitudes of Australian practice nurses in providing nutrition care to patients with chronic disease. Fam Prac, 31, 201-208.

32. Ball, L., Desbrow, B., \& Leveritt, M. (2014). An exploration of individuals' preferences for nutrition care from Australian primary care health professionals. Aust J Prim Health, 20 113-120.

33. Archuleta M, VanLeeuwen D, Halderson K, Wells L, Bock M (2012). Diabetes Cooking Schools Improve Knowledge and Skills in Making Healthful Food Choices. Journal of Extension. 50.

34. Corr, L., Rowe, H., \& Fisher, J. (2015). Mothers' perceptions of primary health-care providers: thematic analysis of responses to open-ended survey questions. Aust J Prim Health, 21 58-65.

35. Sahlsten, M. J., Larsson, I. E., Sjostrom, B., \& Plos, K. A. (2008). An analysis of the concept of patient participation. Nurs Forum, 43, 2-11. 
Table 1: Categories and sub-categories emerging from the interviews.

\begin{tabular}{|c|c|c|}
\hline Categories & Definition & Sub-categories \\
\hline $\begin{array}{l}\text { Receiving directive } \\
\text { nutrition care is } \\
\text { overwhelming and } \\
\text { not always helpful }\end{array}$ & $\begin{array}{l}\text { Initial experience of } \\
\text { having an appointment } \\
\text { with a dietitian and } \\
\text { receiving nutrition care } \\
\text { was viewed as } \\
\text { overwhelming and not } \\
\text { always helpful }\end{array}$ & $\begin{array}{l}\text { - Initial appointment occurs soon after } \\
\text { diagnosis } \\
\text { - } \quad \text { Instructional, directive care is } \\
\text { challenging and overwhelming } \\
\text { - Variable views about the helpfulness } \\
\text { of nutrition care from dietitians }\end{array}$ \\
\hline $\begin{array}{l}\text { Contribution of } \\
\text { dietary guidelines in } \\
\text { supporting dietary } \\
\text { change }\end{array}$ & $\begin{array}{l}\text { Views on the content of } \\
\text { nutrition care, and } \\
\text { appropriateness of } \\
\text { dietary guidelines }\end{array}$ & $\begin{array}{l}\text { - Confusion from conflicting dietary } \\
\text { messages } \\
\text { - } \text { Questioning the relevance of general } \\
\text { guidelines for individual needs } \\
\text { - Supporting healthy dietary intake } \\
\text { beyond the dietary guidelines }\end{array}$ \\
\hline $\begin{array}{l}\text { Desiring more } \\
\text { individualised care }\end{array}$ & $\begin{array}{l}\text { Views on how dietitians } \\
\text { can improve delivery of } \\
\text { nutrition care to } \\
\text { patients }\end{array}$ & $\begin{array}{l}\text { - Feeling rushed and not heard in } \\
\text { consultations } \\
\text { - Wanting genuine relationships and } \\
\text { open communication } \\
\text { - Desiring nutrition care that is tailored } \\
\text { to individual circumstances }\end{array}$ \\
\hline
\end{tabular}

